\title{
Efficacy of the levonorgestrel intrauterine system in treating menorrhagia: Actualities and ambiguities
}

\author{
Amaju Ikomi, MB BS, MRCog, Consultant; Eric F Pepra, MB ChB, Senior House Officer, Department of Obstetrics and \\ Gynaecology, Basildon Hospital, Essex, UK
}

Correspondence. Dr A Ikomi, Department of Obstetrics and Gynaecology, Basildon Hospital, Nethermayne, Basildon, Essex SS16 5NL, UK. Tel: +44 (0) 1268 533911.Fax: +44 (0) 1268 533195.E-mail: maj.ikomi@virgin.net

(Accepted $16^{\text {th }}$ July 2001)

The Journal of Family Planning and Reproductive Health Care 2002: 28(2): 99-100

\begin{abstract}
Objective. To review the literature on usage of the levonorgestrel intrauterine system (LNG IUS) in women with fibroids or adenomyosis in an attempt to develop a pragmatic approach when faced with enlarged uteri.

Data sources. Computerised searches of MEDLINE and EMBASE up to December 2000. Some of the information was obtained from references listed in the primary sources and references already known to the authors.

Study selection. Studies and case reports were excluded if they did not specifically provide information about LNG IUS usage in women with fibroids and adenomyosis.

Data extraction and synthesis. After perusal, each relevant publication was summarised and appraised in terms of whether it contained information relevant to the stated objective.

Discussion. This area is sparsely researched, as there were no randomised-controlled studies. One large contraceptive study suggests the LNG IUS has a protective effect from the complications of uterine fibroids and smaller case studies suggest it is also useful in women with symptomatic fibroids. However, there is also evidence of an increased risk of expulsion and there may be a place for initial adjunctive medical treatment to minimise this risk.

An observational study and isolated reports of women with adenomyosis report significant symptomatic relief in these women.

Conclusion. Available data refute the notion that uterine enlargement is a contraindication to insertion of the $L N G$ IUS, but there appear to be reasons for a cautious approach when dealing with pathological uteri.
\end{abstract}

\section{Key message points}

- The levonorgestrel intrauterine system has been used successfully in women with uterine enlargement due to fibroids and adenomyosis.

- Available evidence points to an increased risk of expulsion associated with significant uterine enlargement or documented anaemia.

- Prospective research studies need to clarify this issue and evaluate possible solutions.

\section{Introduction}

The levonorgestrel intrauterine system (LNG IUS: Mirena $^{\circledR}$, Schering, UK) is a highly effective contraceptive ${ }^{1}$ and is extremely helpful in treating menorrhagia ${ }^{2}$ among other confirmed or highly suggestive non-contraceptive benefits. ${ }^{3}$ Randomised trials have confirmed its superior efficacy when compared with conventional medical treatment for menorrhagia ${ }^{4}$ and its comparability with hysteroscopic surgical ablation of the endometrium. ${ }^{5}$ Its advantages over endometrial ablation are: relative simplicity as well as reversibility, contraceptive effect ${ }^{6}$ and the relief of pelvic pain. ${ }^{7}$ The latter is frequently of even greater importance to the woman herself and is seldom relieved by, for example, transcervical resection of the endometrium (TCRE). ${ }^{8}$ Recently published studies suggest the potential of the IUS to reduce hysterectomy waiting lists, ${ }^{9,10}$ and it is generally accepted as a first option in women with heavy periods who also require contraception.

Available information on the LNG IUS as a treatment for menorrhagia is mainly derived from studies of carefully selected women with dysfunctional uterine bleeding in research settings. However, in routine clinical practice, heavy menstrual loss is often due to underlying uterine pathology, most commonly fibroids and adenomyosis. It is therefore important to consider the possible effect of these conditions on the efficacy of the device and with this in mind, the available literature was reviewed.

\section{Effect of uterine fibroids}

Uterine myomas (fibroids) are benign tumours, which primarily contain smooth muscle, but may have varying densities of fibrous elements. Postmortem studies suggest that up to $50 \%$ of all women have fibroids ${ }^{11}$ and that these are commonly associated with heavy menstrual loss. ${ }^{12}$ This means practitioners who use the LNG IUS will undoubtedly encounter fibroids on a regular basis. Significant enlargement or distortion of uterine cavities, as well as excessive bleeding secondary to fibroids, can potentially affect the positioning of the device and hence efficacy.

In addressing this issue, one also has to consider the possible effect of the released progestogen (levonorgestrel) on existing fibroids. This is a controversial subject as in vitro $^{13}$ and some clinical studies ${ }^{14}$ have suggested a stimulatory effect in certain circumstances of some progestogens on uterine fibroids. Long-term contraceptive studies, however, have demonstrated protection from the complications of fibroids in association with use of depotmedroxyprogesterone acetate (DMPA) ${ }^{15}$ and the LNG IUS $^{7}$. Intramuscular DMPA causes moderately high systemic levels of the progestogen medroxyprogesterone and results in ovarian and endometrial suppression. In marked contrast, the LNG IUS causes selective endometrial suppression due to high endometrial and very low systemic concentration of the progestogen levonorgestrel, with very little effect on ovarian function. ${ }^{16}$ The apparent protective effect of these two forms of contraception, despite different 
systemic levels of progestogen, has led some to hypothesise that the main mechanism of this protection may be their common effect of endometrial suppression. ${ }^{17}$ This hypothesis is supported by the fact that some endometrial growth factors have been shown to be mitogenic for myoma cells in culture. ${ }^{18}$

Although encouraging, this protective effect cannot simply be extrapolated to women with existing symptomatic fibroids, and separate studies are needed.

A pilot study of five menorrhagic LNG IUS users with fibroids showed a reduction in uterine volume after 6-18 months of use ${ }^{19}$ and a similar effect is documented in isolated reports. ${ }^{20}$ One retrospective study did not demonstrate an adverse effect on overall success rates due to fibroids. ${ }^{21}$ However, a less reassuring finding in this study was the fact that all expulsions of the device were associated with the presence of uterine fibroids and the factors that significantly contributed to this association were major uterine enlargement (volume $>148 \mathrm{ml}$ ) and pretreatment anaemia. The deductions were that uterine enlargement can affect positioning of the device and anaemia is indicative of truly excessive blood loss, which is more likely to dislodge the device. This study also reported that in the majority of women who required hysterectomy following failure with the LNG IUS, histological examination of the uterine specimen confirmed the presence of fibroids. Reports of prolapsed pedunculated fibroids following insertion of the LNG IUS ${ }^{22}$ lend further support to a recommendation for extra vigilance when dealing with fibroid uteri.

Although some of the available evidence does raise concern, published studies on LNG IUS usage in menorrhagic women do include some women with fibroids, and successful outcomes have been reported. Therefore, it would be simplistic to try and base any recommendations for selection only on the presence or absence of fibroids, as this will simply deny many potential beneficiaries. Nevertheless, the fact remains that no one has specifically addressed, in well-designed prospective trials, the issue of how their presence affects the efficacy of the device. Thus there is a lack of clear guidelines about LNG IUS usage in women with fibroid uteri. If prospective studies do confirm an adverse relationship, other important considerations are whether there is a critical uterine size and whether alternative approaches, such as initial adjunctive treatment with other suppressive agents such as GNRH analogues, make a difference. The reasoning behind this assertion is the fact that expulsion of the LNG IUS (like all intra-uterine devices) is most common during heavy menstrual loss, particularly the first and second such heavy loss after insertion. It has been reported that $40 \%$ of women with objective menstrual loss in excess of $200 \mathrm{ml}$ have uterine fibroids, whereas only $10 \%$ of those with a loss of $80-100$ $\mathrm{ml}$ are affected. ${ }^{23}$ In view of the association between fibroids and heavy menstrual flow, it might well be beneficial to abolish the first one or two menses during early use of the LNG IUS in women with fibroids.

\section{Effect of adenomyosis}

The existence of endometrial tissue within the myometrium leads to heavy and usually painful menstrual loss. Diagnosis has traditionally relied on clinical findings with histological confirmation following hysterectomy, as conventional medical treatment tends to be unhelpful. Major developments include an ability to detect this condition using MRI scans, and studies have confirmed the LNG IUS to be an efficacious method of treating the symptoms of this condition. ${ }^{24}$ In this prospective study the use of LNG IUS in patients with adenomyosis associated menorrhagia led to symptomatic relief in $92 \%$ of the patients after 1 year follow-up. Significant increases in serum haemoglobin and ferritin were also observed.

A more recent case report described a significant reduction in of a very large adenomyotic uterus associated with use of the LNG IUS. ${ }^{25}$ This suggests a potential role in the treatment of adenomyosis irrespective of uterine size, but further studies are needed.

\section{Conclusions}

Practitioners increasingly see the LNG IUS as a panacea to the problem of excessive menstrual loss, and in Western European countries outside Scandinavia, formal licenses for treating heavy periods are anticipated. In the continuing absence of relevant prospective studies of women with pathology-related menorrhagia, and based on the currently available evidence, one concludes by suggesting a cautious approach.

When fibroids or adenomyosis are suspected, routine initial ultrasound scan of the uterus should be combined with sonohysterography or hysteroscopy to check the contours of the uterine cavity. Cavity distortion precludes insertion of the device. Significant uterine enlargement or documented anaemia may increase the risk of expulsion, and under these circumstances there may be scope for initial adjunctive treatment as well as suitable counselling of the woman.

\section{Statements on funding and competing interests}

Funding. None declared.

Competing interests. None declared.

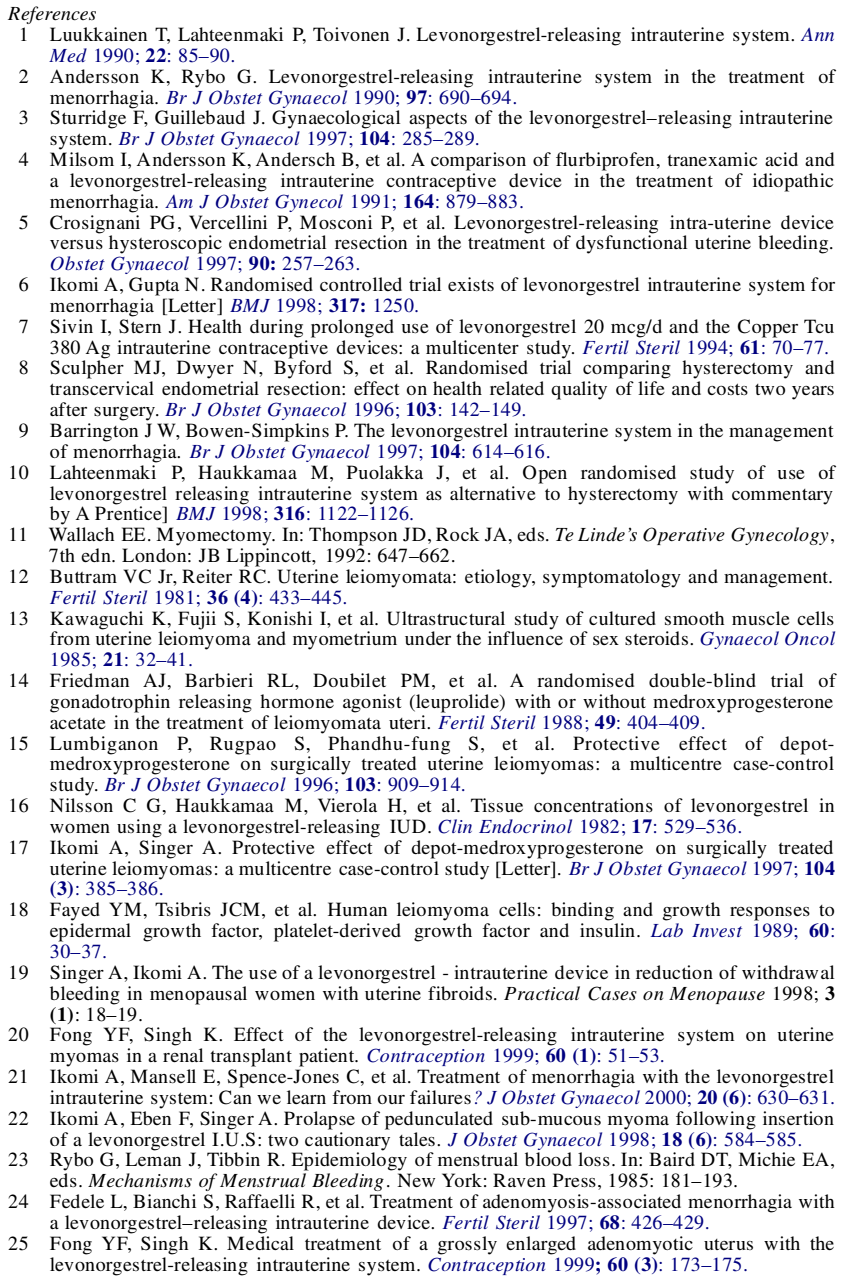

( Gy Milsom I, Andersson K, Andersch B, et al. A comparison of flurbiprofen, tranexamic acid and J

Crosignani PG, Vercellini P, Mosconi P, et al. Levonorgestrel-relesing intratuterine device ersus hysteroscopic endometrial resection in the treatment of dysfunctional uterine bleeding. r] BMJ 1998; 317: 1250

Stern J. Health during prolonged use of culpher MJ, Dwyer N, Byford S, et a multicenter study. Fertil Steril 1994; 61: 70-77. quality of life and costs two years

Lahteenmaki P, Hauk a $M$. Puolakk J, et al Open randomised study of use of evonorgestrel releasing intrauterine system as alternative to hysterectomy with commentary

The

. Kawaguchi K, Fujii S, Konishi I, et al. Ultrastructural study of cultured smooth muscle cells rom uterine leio

A randomised double-blind trial of acetate in the treatment of leiomyomata uteri. Fertil Steril 1988; 49: 404-409. Lumbiganon $\mathrm{P}$, Rugpao S, Phandhu-fung $\mathrm{S}$, et al. Protective effect of depot-

ilsson C G, Haukkamaa M, Vierola H, et al. Tissue concentrations of levonorgestrel in gestrel-releasing IUD. Clin Endocrinol 1982; 17 ;

Fayed YM, Tsibris JCM, et al. Human leiomyoma cells: binding and growth responses to $30-37$ 1): $18-$

. komi A, Mansell E, Spence-Jones C, et al. Treatment of menorrhagia with the levonorgestre intrauterine system: Can we learn from our failures? J Obstet Gyndecol

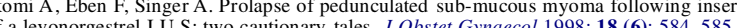
Ry G, Leman J, Tibbin R. Epidemiology of menstrual blood loss. In: Baird DT, Michie EA, eds. Mechanisms of Menstrual Bleeding. New York: Raven Press, 1985: 181-193. a levonorgestrel-releasing intrauterine device. Fertil Steril 1997; 68: 426-429. evonorgestrel-releasing intrauterine system. Contraception 1999; 60 (3): 173-175. 